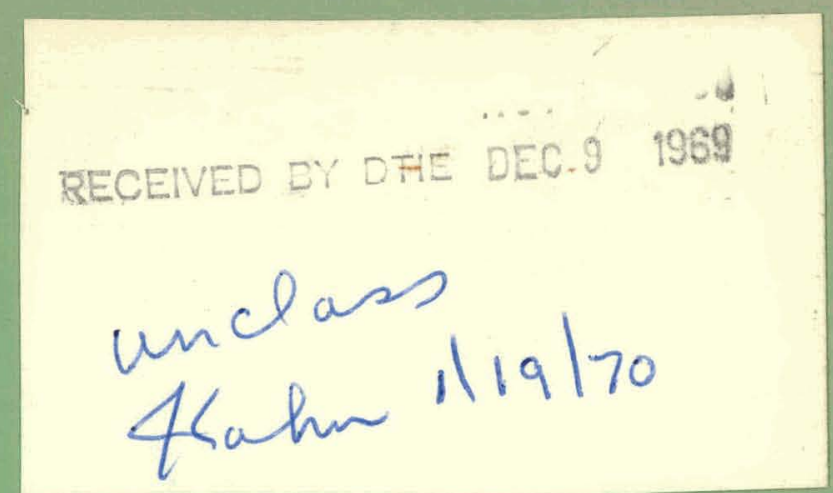

\title{
LASER TECHNIQUES FOR APPLICATION \\ TO SURFACE TOPOGRAPHY AND ROUGHNESS \\ MEASUREMENTS
}

Gerald B. Brandt

December 18, 1967

\section{Westinghouse Research Laboratories}

PITTSBURGH, PENNSYLVANIA 15235

Pisosian) 


\section{DISCLAIMER}

This report was prepared as an account of work sponsored by an agency of the United States Government. Neither the United States Government nor any agency Thereof, nor any of their employees, makes any warranty, express or implied, or assumes any legal liability or responsibility for the accuracy, completeness, or usefulness of any information, apparatus, product, or process disclosed, or represents that its use would not infringe privately owned rights. Reference herein to any specific commercial product, process, or service by trade name, trademark, manufacturer, or otherwise does not necessarily constitute or imply its endorsement, recommendation, or favoring by the United States Government or any agency thereof. The views and opinions of authors expressed herein do not necessarily state or reflect those of the United States Government or any agency thereof. 


\section{DISCLAIMER}

Portions of this document may be illegible in electronic image products. Images are produced from the best available original document. 


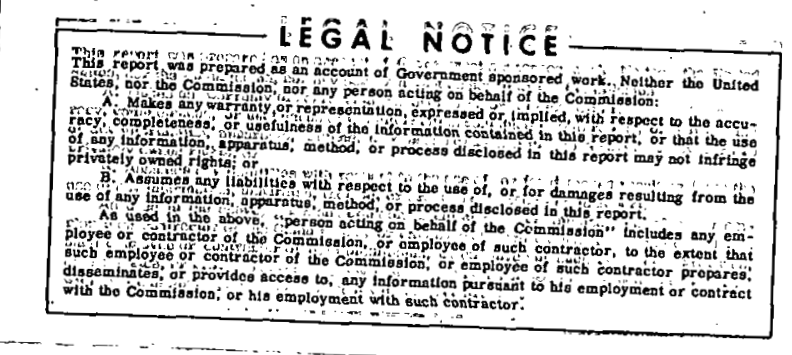

WERL-HOLOG-1

Q

LASER TECHNIQUES FOR APPLICATION

TO SURFACE TOPOGRAPHY AND ROUGHNESS

MEASUREMENTS

Gerald B. Brandt

Quantum Electronics R\&D

December 18, 1967 


\section{ABSTRACT}

Four laser techniques have been developed for the study of the topography and surface roughness of simulated corrosion sample surfaces. Two methods using holographic contour generation provide depth information with approximately 10 micron resolution. Two laser scattering experiments provide roughness data. Experimental results and recommendations for further research are presented. 
LASER TECHNIQUES FOR APPLICATION TO SURFACE TOPOGRAPHY AND ROUGHNESS MEASUREMENTS

\section{G.B. Brandt}

The purpose of this investigation was to develop holographic and coherent optical techniques suitable for the study and measurement of surface thickness variations, topography, and roughness. To these ends, a 6 month investigation was undertaken to study the feasibility of using the following techniques: (1) Holographic recording of simulated corrosion surfaces under magnification and height measurement from reconstructions from these holograms. (2) Application of multiple beam interferometry to the measurement of surface topography. (3) Measurement and interpretation of the properties of the diffraction pattern produced when a rough surface is illuminated by laser light. This report summarizes the results of the investigation and includes recommendation for further study.

HOLOGRAPHIC RECORDING

A hologram "microscope" shown schematically in Figure 1 was set up on a large spring mounted granite table for study of simulared corrosion samples. Using approximately $100 \mathrm{mw}$ of single mode argon laser illumination at $4880 \AA$, holograms were made of these surfaces through the microscope objective and were reconstructed and photographed by the camera. At approximately $21 \mathrm{X}$ magnification, the resolution of the hologram recorded image was as good as permitted by the quality of the optical system which was used. Photographs of the hologram reconstruction and the direct image through the microscope were indistinguishable in regard to resolution and sharpness at the 21X magnfication used (see Figure 2). 
Dwg. $854 A 452$

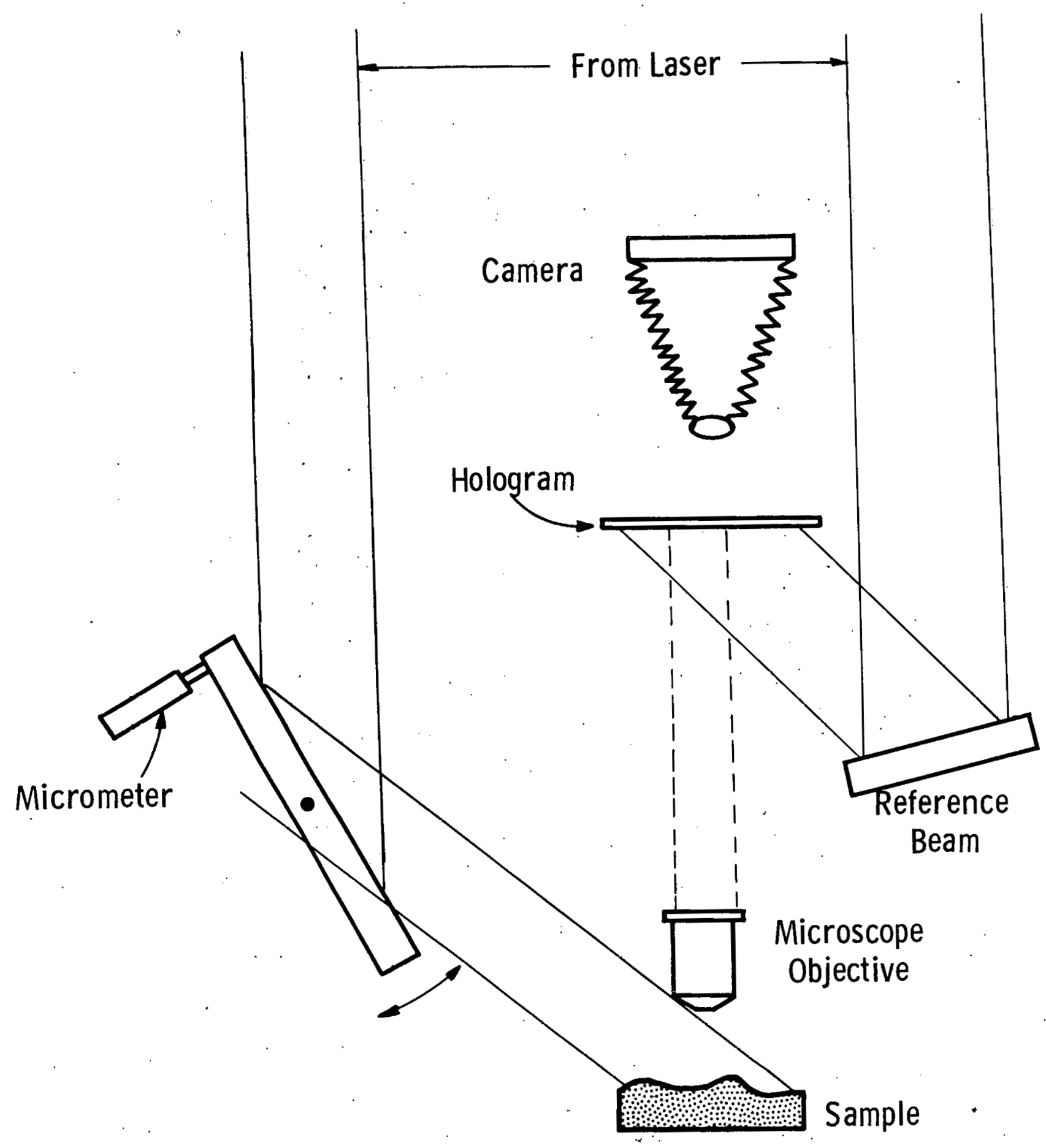

- FIGURE 1. Schematic of Experimental Arrangement for Hologram Microphotography and Contour Generation by Moving Source Method 


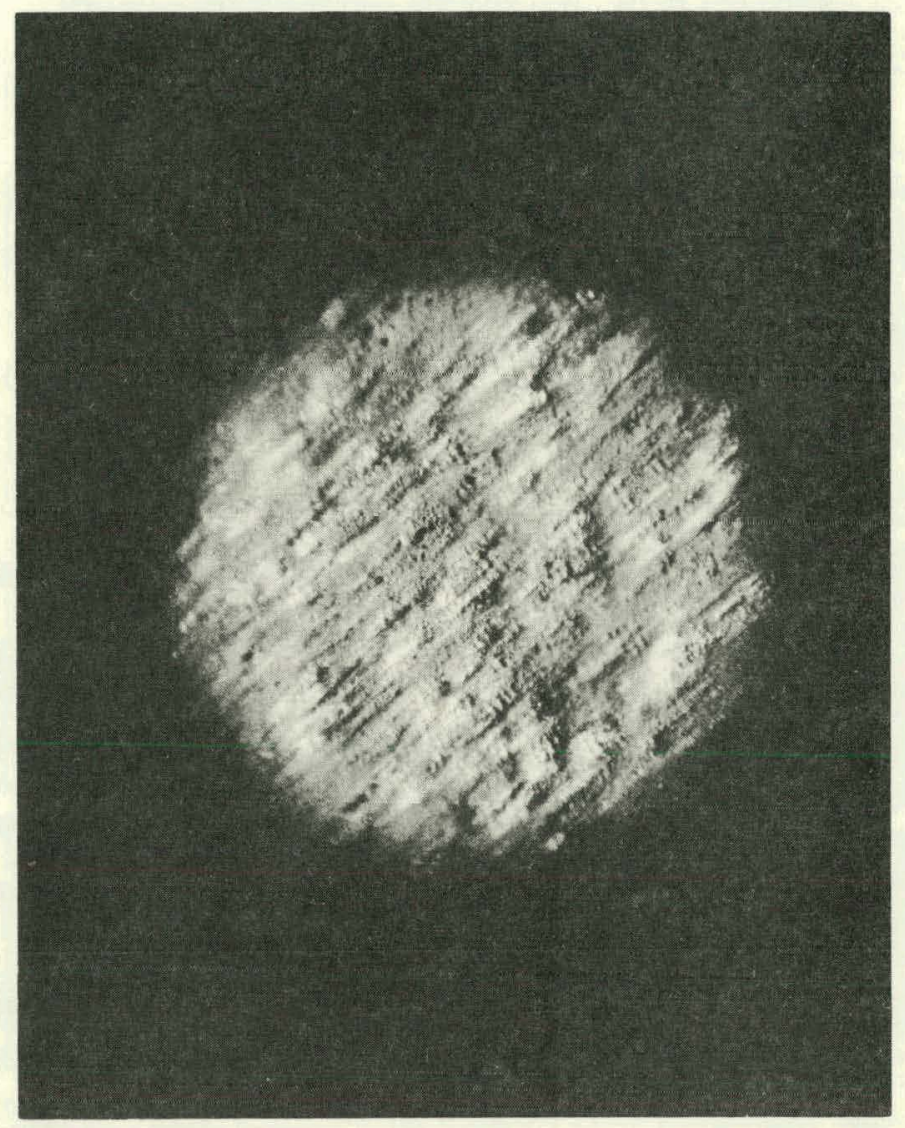

FIGURE 2. Photograph of Simulated Corrosion Sample Surface (21X). 
The possibility of using the hologram reconstruction directly to measure the heights of features on the surface was considered. Since the lateral and axial magnifications of the reconstructed three dimensional surfaces differ by a factor of $\mathrm{m}$, where $\mathrm{m}$ is the magnification of the microscope objective, a qualitative measure of the heights of different portions of the surface can be found by focussing an eyepiece on different portions of the surface and measuring the position of the eyepiece relative to the point of best focus of another portion of the surface. Height changes are magnified by a factor of $\mathrm{m}^{2}$ and thus are of the proper order of magnitude for mechanical measurement. The difficulty with this means of height measurement is that it is inherently a point by point measurement whose accuracy depends upon a subjective judgment regarding the point of best focus of the image. Since the corrosion sample surfaces are relatively featureless, this point of focus is often difficult to determine. Although automatic focussing devices might improve the accuracy of this method, more satisfactory means of retrieving the height information were developed and are discussed below.

Hologram recording of the simulated corrosion surfaces presented no particular technical difficulty. The requirements for the stability of the source and reference beam were easily met; in a working environment where vibration of the sample is encountered, pulsed laser holography could be employed. The expected advantage of high depth of field in the reconstruction was achieved and it appears that the resolution of a hologram reconstructed with a replica of the original reference beam is more than adequate for the present application.

HOLOGRAM CONTOUR GENERATION

Whereas holography provides a solution to the problem of recording a three dimensional volume with all portions equally in focus, the difficulty in retrieving the depth information remains even when a three dimensional record is available. One goal of the 
investigation is the production of a topographic map of the surface from which measurements of the thickness of a deposited layer can be made. Two methods of double exposure holography ${ }^{1,2}$ which produce reconstructions with interference fringes whose spacing contains information about the height changes on the surface, were investigated.

A technique which produces contour fringes on the reconstruction was developed by Tsuruta and his co-workers. ${ }^{2}$ This technique, which is analogous to contour study by the method of replicas, ${ }^{3}$ involves making a double exposure hologram of the sample immersed first in a liquid of index of refraction $n_{1}$ and second in a liquid of index $\mathrm{n}_{2}$. The resulting hologram reconstruction is crossed by fringes representing loci of constant thickness between the sample and the cell window. For small index differences and normal illumination the contour interval is given by

$$
\Delta \mathrm{h}=\lambda / \Delta \mathrm{n}(1+\cos \theta)
$$

where $\Delta \mathrm{n}=\mathrm{n}_{1}-\mathrm{n}_{2}$ and $\theta$ is the angle between the surface normal and the viewing direction. For zero illumination and viewing angles Equation 3 becomes

$$
\Delta \mathrm{h}=\lambda / 2 \Delta \mathrm{n}
$$

Realizable contour intervals for various liquids compared with water appear in Table $I$.

TABLE 1

Contour Interval Contour Interval Liquid Index $\Delta \mathrm{n}$ (rel to water) $\lambda=.488$ Microns in Wavelengths

\begin{tabular}{lrrrrr}
\hline Water & 1.3330 & - & - & \\
Methano1 & 1.3312 & .0018 & 135 microns & $278 \lambda$ \\
Acetone & 1.3589 & .0259 & 9.4 microns & $19.3 \lambda$ \\
Ethanol & 1.3624 & .0294 & 8.3 microns & $17.0 \lambda$ \\
Trichloroethylene & 1.4777 & .1447 & 1.7 microns & $3.5 \lambda$
\end{tabular}


Dwg. 854A453

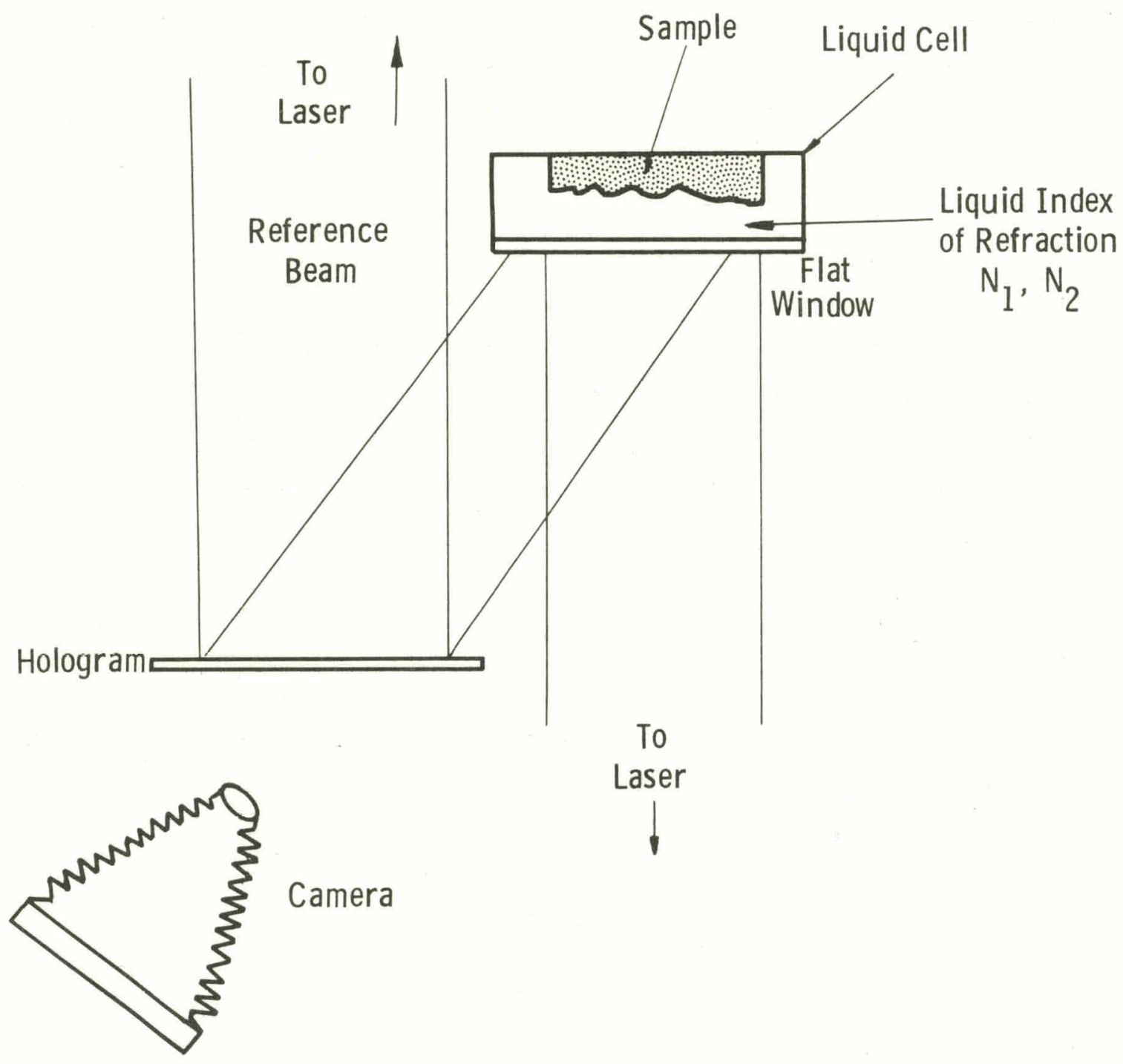

FIGURE 3. Schematic of Experimental Arrangement for Contour Generation by the Immersion Method 


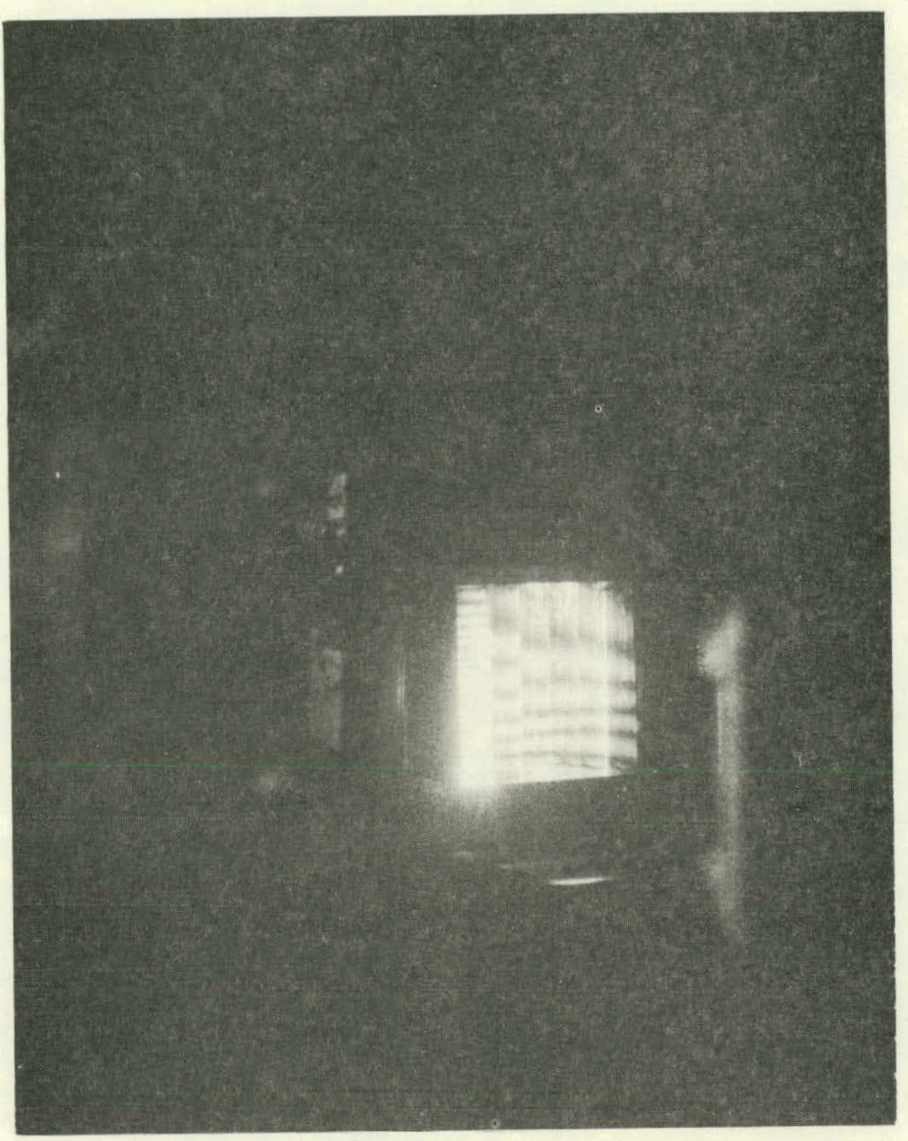

FIGURE 4. Hologram Generated Cnntours on Wedged Test Plate. 
In order to verify the predictions of Equations 1 and 2 the experimental arrangement shown in Figure 3 was used to generate contours on the reconstruction of a 2 by 2 inch aluminum plate with a series of wedges milled on the face. The results of a double exposure using water and methanol are shown in Figure 4. Here the contour interval is 135 microns per fringe in the vertical direction. The wedge on the left has 21 fringes crossing it indicating a thickness change from one end to the other of .111 inches. Since the wedge milled on the aluminum block measures .100 inches difference in thickness from one end to the other, a tilt of .011 inches between the block and the window is indicated. Unless the immersion technique can be used with some magnification, it is not applicable to the corrosion analysis problem. Attempts to use the arrangement in Figure 3 with a microscope objective to enlarge the image of the surface were unsuccessful for index of refraction differences of .03 or greater. The reason is that the image defocuses as the immersion liquid is changed thus if interference fringes are formed, they do not form in the image plane. This limitation can be circumvented by the construction of a double walled cell and the interchange of the two liquids between the two parts of the cell rather than simply the replacement of one liquid. If such a cell were built, contouring could be done at much higher magnification that was achieved in the present work. The advantage of the immersion technique is that contours correspond to height changes normal to the surface, and no correction is needed for tilt as in the case of the doublt exposure contour generation experiments. In one sense, the ability of the hologram to provide height information directly on the reconstructed image is its most important contribution to the solution of the corrosion inspection problem. Hildebrand and Haines ${ }^{1}$ were the first investigators to point out the possibility of utilizing multiple exposure holography to generate interference fringes on the reconstruction which represent loci of constant "range" from the hologram plane. They investigated two techniques, multiple wavelength (double exposure at separate laser wavelengths) and multiple source, (either double exposure--moving the 
apparent illuminating source between exposures, or single exposure with two sources illuminating the sample.) The second of these techniques is the simplest and was the one chosen for application to the contouring problem.* If a double exposure hologram is made of a surface with the angle of illumination changed by a small angle $\Delta \theta$ between exposures, providing that the illumination is paralle1 to the surface and that the reconstruction is viewed perpendicular to the surface, the reconstruction will be crossed by fringes of constant height whose spacing is given by

$$
\Delta \mathrm{h}=\lambda /(2 \sin (\Delta \theta / 2))
$$

where $\lambda$ is the laser wavelength. If the illumination is not parallel to the surface, the fringes represent path length differences of a laser wavelength between the two sources and the observer. In a sense the fringes for oblique illumination can be thought of as contours on a steep hill. The general expression for fringe formation is

$$
r_{1}-r_{2}=n \lambda
$$

where $r_{1}$ and $r_{2}$ are the distances from the two sources to the object and $\mathrm{n}$ is an integer. The principle drawback of this method is the requirement for the illumination to be parallel to the surface of the sample in order to obtain true contours of the surface.

For illumination which is not parallel to the surface under study, a straightforward geometrical calculation provides the formulas for interpretation of the fringe system in terms of height changes. If $d_{0}$ is the spacing of the fringes which would be produced by two laser sources at an angle $\Delta 0$, namely

$$
\mathrm{d}_{0}=\lambda / 2 \sin (\Delta \theta / 2)
$$

\footnotetext{
For a recent improvement on the first method--see Ref. 11.
} 
then the spacing $d$ of the fringes which will be observed on a reconstruction looking perpendicular to a surface is

$$
\mathrm{d}=\left(\mathrm{d}_{0} / \cos \theta\right) \quad\left\{1-\left(\mathrm{h} / \mathrm{d}_{\mathrm{o}}\right) \sin \theta\right\}
$$

where $h$ is the height change of the surface per fringe and $\theta$ is the angle of illumination with respect to the direction perpendicular to the surface. Clearly $h$ per fringe is dependent upon the slope, $\tan \phi$ abbreviated as $\mathrm{S}$. The height change per fringe is

$$
\mathrm{h}=\mathrm{s} \mathrm{d}_{\mathrm{o}} /(\cos \theta+\mathrm{s} \sin \theta)=\mathrm{d}_{\mathrm{o}} /(\cot \phi \cos \theta+\sin \theta) .
$$

For $\phi=\theta$ Equation 7 reduces to Equation 3. Equation 6 is the more useful of the above equations; from it, knowing the distance between fringes on the hologram reconstruction and knowing the angle of illumination, the laser wavelength, and the angle $\Delta \theta$ through which the illuminating surface can be calculated. Once the height change is calculated from $d / d_{0}$ in Equation 6 then the average slope can be calculated from Equation 7. Including a standard wedge in an initial photograph would allow direct calibration of the fringe system independent of the geometrical calibration.

Because of the defocusing effect of the immersion technique at magnifications greater than about $3 \mathrm{x}$, the investigation was shifted to the contour generation technique. This technique may be thought of as a projection on the sample surface of a series of straight fringes of spacing given by Equation 6 produced by the interference between two coherent sources. Using the experimental arrangement in Figure 1 holograms were made of a simulated corrosion deposit layer and reconstructed at a total magnification of 21X. Figure 5 shows the result of generating contours on a hologram reconstruction by moving the mirror used to illuminate the object by a small angle between exposures of the hologram. Contour intervals are 150, 75, 50, and 37.5 microns per fringe. Because the illumination was approximately 60 degrees from the normal to the surface, the major trend of the 


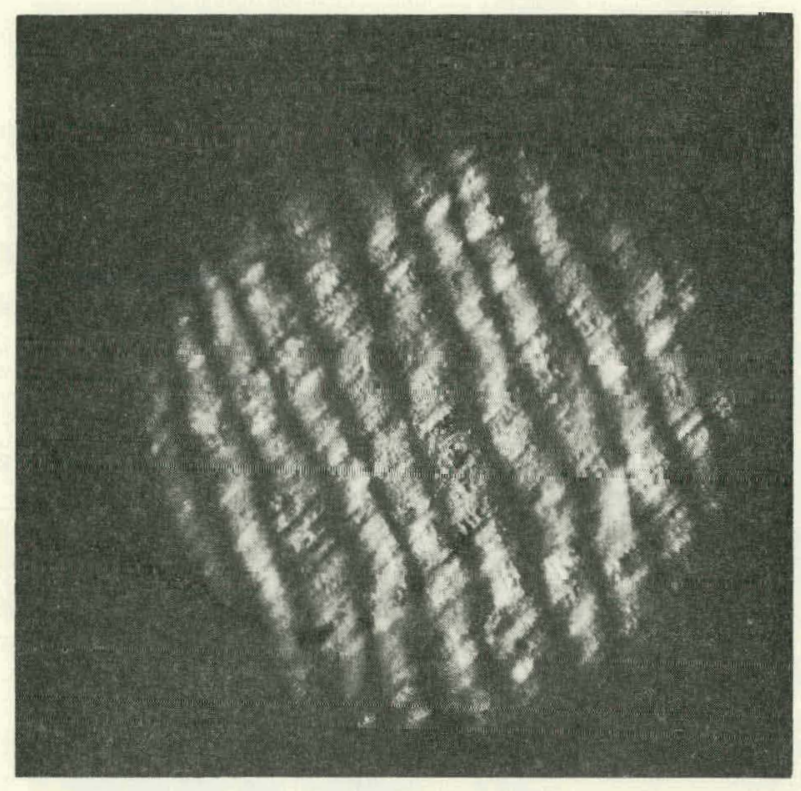

(a)

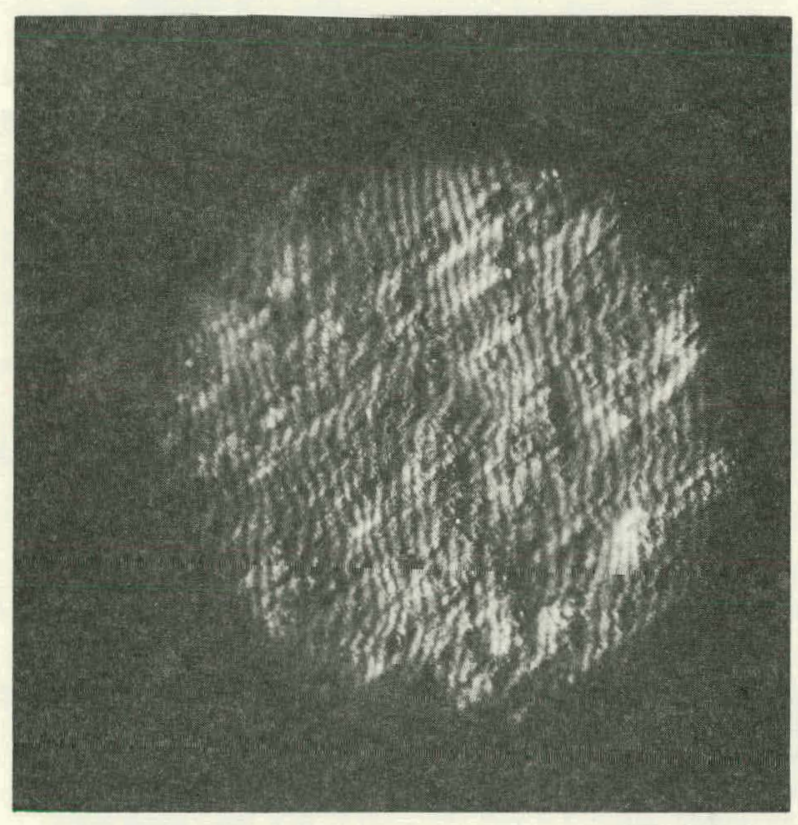

(c)

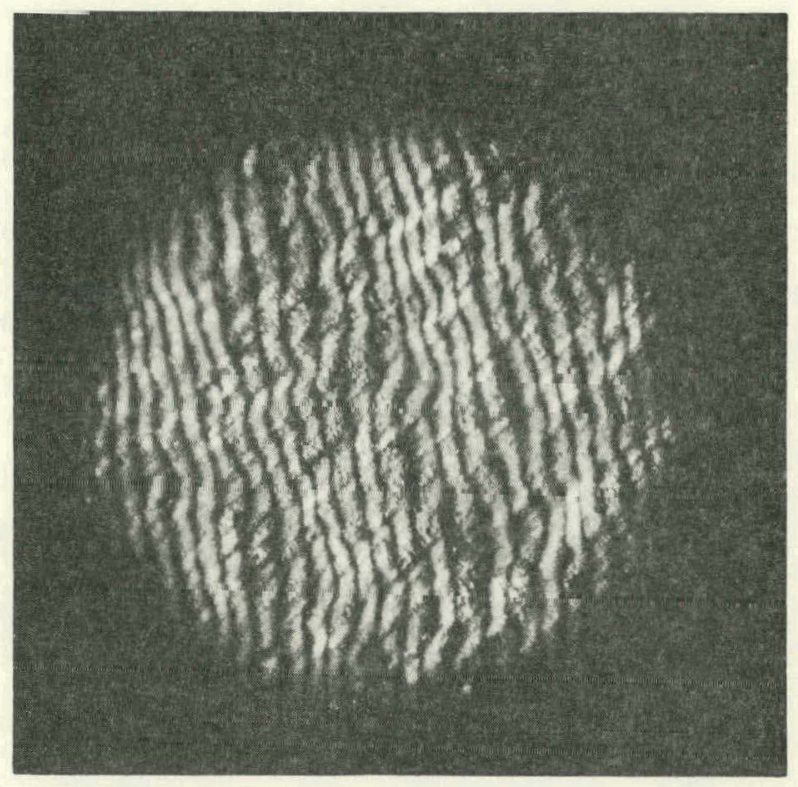

(b)

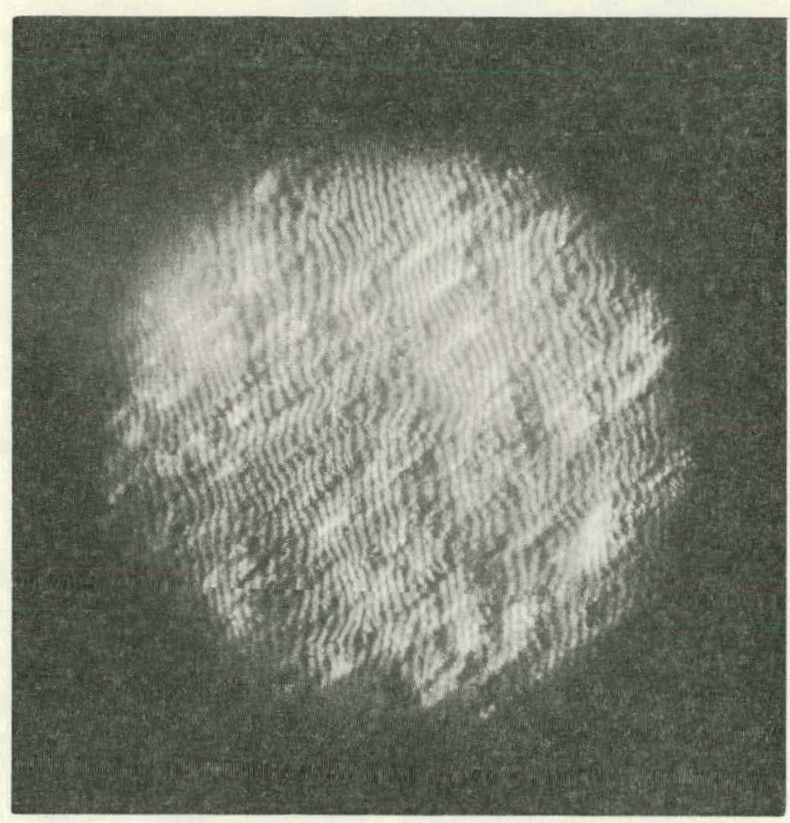

(d)

FIGURE 5. Reconstructions of a Deposited Corrosion Product Layer with Contour Interval (a) 150 Microns,

(b) 75 Microns, (c) 50 Microns, and (d) 37.5 Microns Per Fringe. 
contour fringes is vertical. (The tilt in the fringes in $5 \mathrm{a}$ was probably caused by a slight tilt of the mirror axis as it was rotated.) As the contour intervals become smaller, the details of the surface become more evident. These fringes are interpreted much as one would interpret the contour map of a steep hill, whose maximum slope runs in the horizontal direction in the photographs. By counting the numbers and fractions of fringes crossed along any straight line on the photograph, a cross section of a cut through the surface along that line can be constructed.

It is possible to study the reconstruction from holograms such as those used in Figure 5 directly with an eyepiece and focus on different portions of the reconstructed image to determine which parts of the image correspond to hills and which to valleys. Thus any ambiguity regarding the slope of a surface can be resolved by studying the hologram reconstruction directly. It is to be emphasized that the contour interval is determined by the amount of mirror rotation made between exposures, the angle of illumination, and the laser wavelength. Thus once a given system has been set up and the thenretical predictions for it have been verified, no further calibration is necessary.

\section{MULTIPLE BEAM INTERFEROMETRY}

Multiple beam interferometry has undergone a great deal of development by Tolansky. ${ }^{4}$ This technique involves the establishment of an interference fringe system between two plates, one of which is an optical flat, and the other of which is the sample whose surface topography is desired. The major requirement for the success of multiple beam interferometry is that the test surface and reference flat be highly reflecting. The fringe system which is set up between these surfaces represents height changes on the sample on the order of a wavelength of the illuminating radiation. For height changes which are large with respect to a wavelength, these fringes become very closely spaced, and if the illuminating light is of limited coherence, their contrast decreases. Although it is not treated 
explicitly by Tolansky, reduction of the spatial coherence of the light source by a rough surface will decrease the contrast of the fringes.

Multiple beam interferometry was attempted experimentally with the simulated corrosion samples. An optical flat of approximately $50 \%$ transmission was placed against the sample surface and the region was illuminated with parallel laser light. The area was examined with a microscope for the existence of interference fringes. No fringes were observed with these samples, whereas fringes were observed when the sample was replaced by a mirror. Similar results were obtained with an optical arrangement similar to the Linnik interference microscope. ${ }^{3}$ The conclusion is that the surfaces are not reflective enough to produce good interference fringes. Furthermore, the scattering and diffraction by the roughness of these surfaces destroys the spatial coherence of the laser light thus making the contrast of the fringes extremely low. The surfaces which were examined by this technique were of such coarse topography that the fringe pattern which would have been observed with silvered surfaces would probably have been too fine for easy interpretation. Where the surfaces under investigation are relatively smooth, coating with a highly reflective silver coating allows the study of surface topography by this means.

SCATTERING AND DIFFRACTION OF LASER LIGHT FROM SIMULATED CORROSION

For some time since the availability of lasers, the fact that laser light is diffracted by rough surfaces into a complex pattern called a speckle pattern has been known. ${ }^{5}$ Calculations have been made of the amount of light scattered at different angles from rough surfaces of various statistical properties. ${ }^{6}$ Since these calculations were inspired by the necessity of calculating radar returns from rough bodies they concentrated on calculating the total energy scattered into a given angular region as a function of the polarization and angle of incidence of the optical or radio wavefront.

The second part of the surface inspection problem involves the determination of RMS roughness. Other statistical properties 
such as the average distance between features on the surface and the distribution of slopes on the surface might also be of interest providing that they could be measured in a straightforward manner. Part of the work statement for this IWR included an investigation of the scattering properties of laser light from rough surfaces in order to determine whether roughness measurements can be made by this means. Studies were made of the light diffracted from three "standard" lapped surfaces of niminal roughness of 2, 4 and 8 microinches. ${ }^{7}$ Figure 6 shows microphotographs of these three surfaces at about 50X magnification. Each small division on the reticule corresponds to approximately 20 microns. Note that the features of all three of the samples have similar general appearance as would be expected from the fact that they are all electroformed replicas of lapped surfaces. Of course the scratches on the roughest surface stand out the most because of the enhanced light scattering by the rough edges. The difficulty in obtaining quantitative roughness measurements from photos of this type can be appreciated.

Two features of the scattering of coherent light were studied to determine their applicability for determination of surface roughness, namely, the amplitude of the scattered light as a function of angle away from the specular direction and the spatial frequency spectrum of the diffraction pattern formed by the coherent light. Both of these studies used the arrangement shown in Figure 7. A collimated laser beam at $4880 \AA$ is focused in one direction by a cylindrical lens on to the surface to be studied. In the other direction, the beam is apertured to a length of $0.5 \mathrm{~cm}$. At a distance of $30 \mathrm{~cm}$. in the specular direction ( $45^{\circ}$ to the sample surface) a recording is made of the reflected light on Polaroid type $55 \mathrm{P} / \mathrm{N}$ film. Typical recordings are shown in Figure 8. Due to the fact that the light has been focused in one direction, the diffraction pattern is spread out in the horizontal plane. In the vertical direction, the light distribution is determined by the scattering properties of the surface. If the sample were perfectly smooth, the reflected light distribution would 


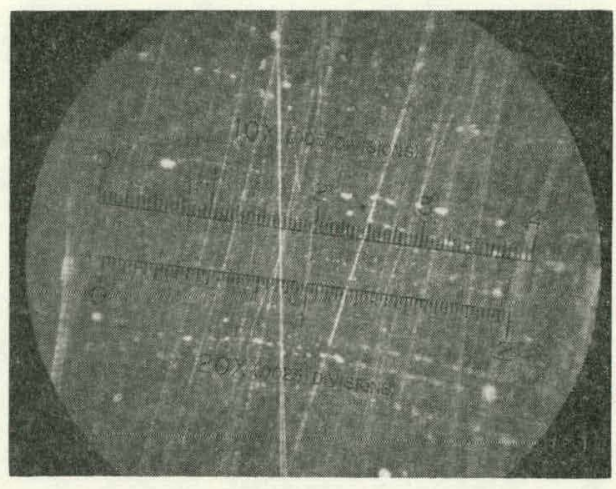

(a)

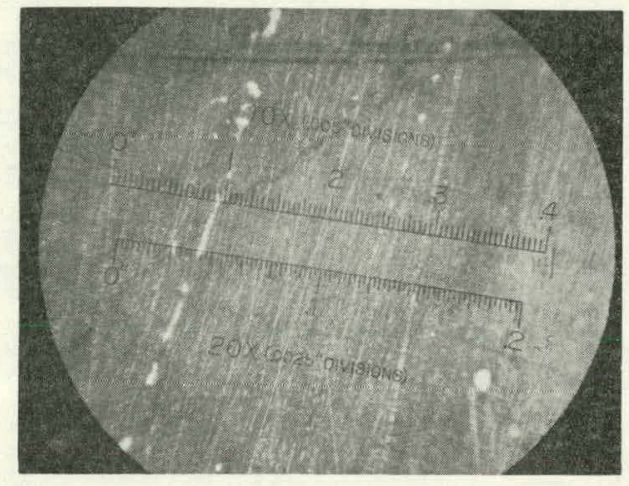

(b)

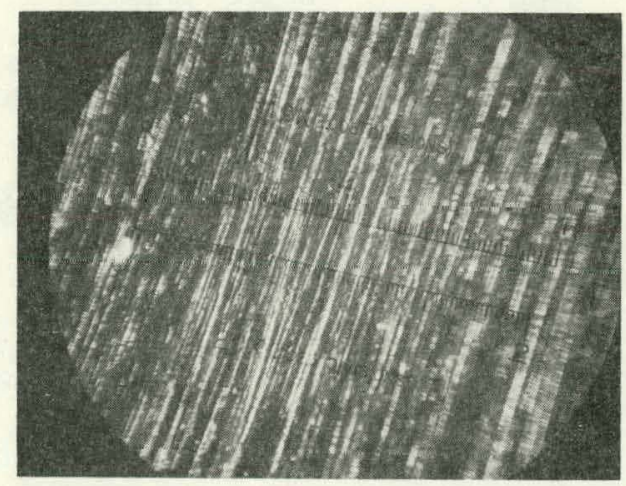

(c)

FIGURE 6. Microphotographs of Lapped Test Surfaces Used in Scattering Experiments. (a) 2 Microinch (b) 4 Microinch (c) 8 Microinch RMS Roughness. 
Dwg. $855 A 150$

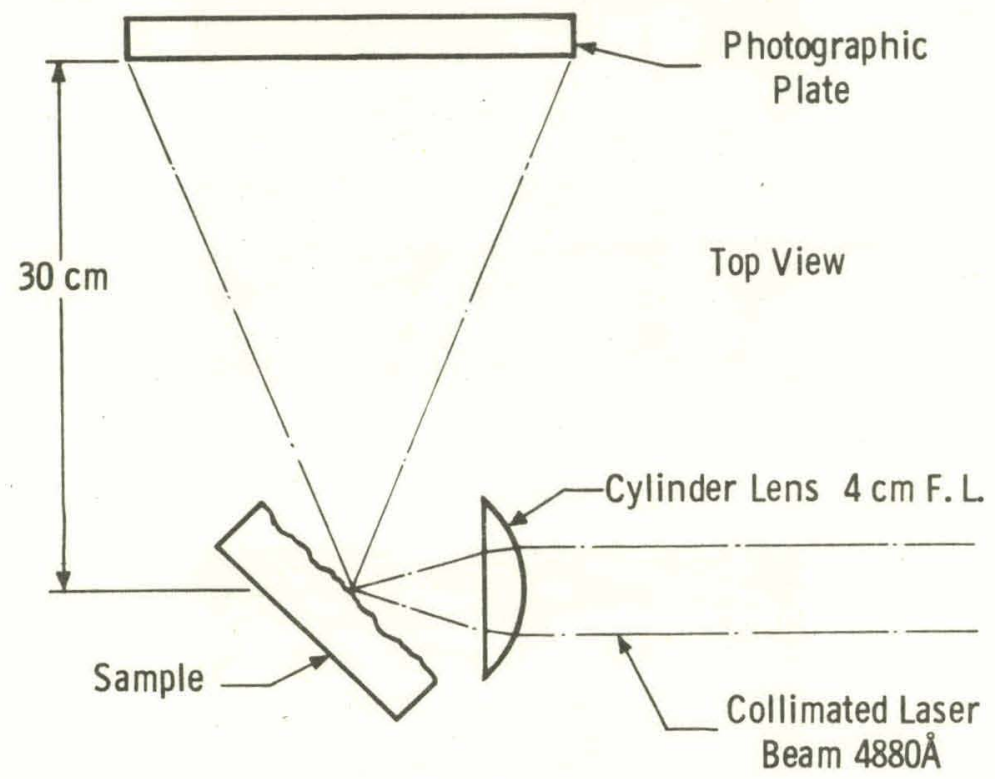

View A

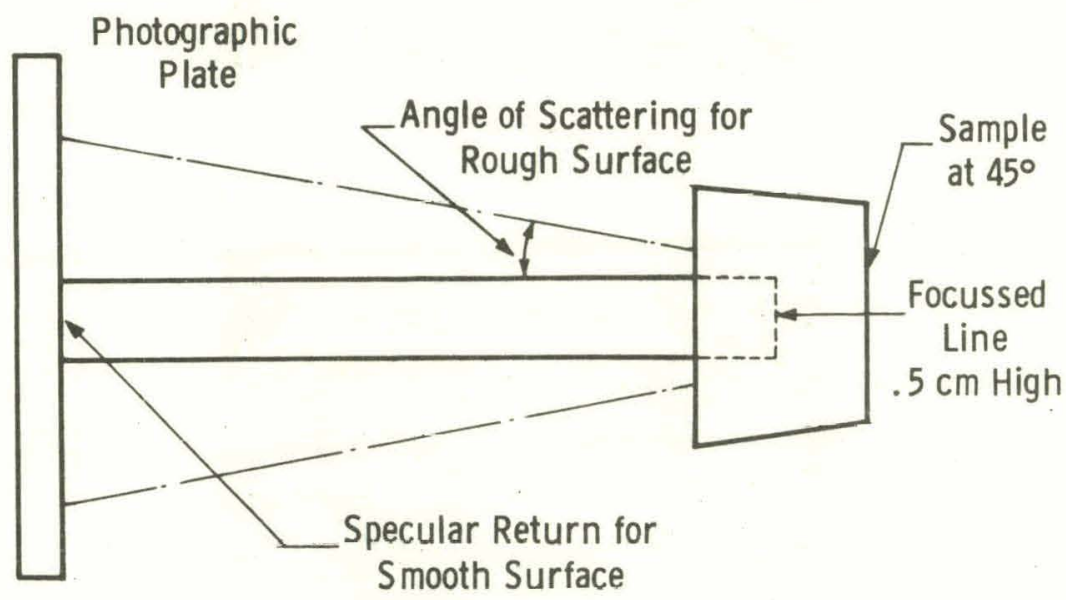

FIGURE 7. Experimental Arrangement for Laser Scattering Measurements--Top View and Side View 
consist of a constant intensity region of height equal to that of the illuminated portion of the sample, with diffraction appearing around the edges. Because the surfaces are rough, light is diffracted into angles away from the direct specular return. From the photographs of the three known samples that of the simulated corrosion surface in Figure 8 it is clear that the amount of light scattered away from specular is a direct function of the roughness of the surface. In order to measure this dependence quantitatively, the densities of the magatives for the photo Figures $8 a-c$ were measured with a scanning densitometer in a direction perpendicular to the direction of the diffracted lines on the photos. The simulated corrosion surface photo was not scanned since the intensity of the pattern appeared to be essentially constant over the diameter of the film. The smoothed results of these scans which were taken at a high scan rate to average out the fluctuations caused by the diffraction pattern, are shown in Figure 9. In this plot, the amplitude of the densitometer output which is proportional to the photographic density of the recording is plotted as a function of distance in $\mathrm{cm}$ from the center of the specular return. Distances can be converted to angle of scattering from specular by dividing by $30 \mathrm{~cm}$, the distance of the film from the sample. The maximum angle of scattering is therefore $1 / 6$ radian or about 10 degress. Although the sample was at an angle of $45^{\circ}$ to the beam in one plane, the measurements are made in a plane perpendicular to the plane of reflection; therefore, Llie appropriate scattering angle to put in the formulas for the scattering as a function of angle is the difference angle from zero. In Beckmann and Spizzichino the formulas for scattering as a function of the geometrical and statistical properties of the surface have been worked out. The intensity of the scattered radiation as a function of the RMS roughness $\sigma$, the correlation distance $T$ of the surface, and the duyle of scattering $\Delta \theta$ measured with respect to the specular direction, is proportional to

$$
I_{\text {scatt }} \sim(T / \sigma) \exp -\frac{\Delta \theta^{2}}{16}\left(\frac{T}{\sigma}\right)^{2}
$$




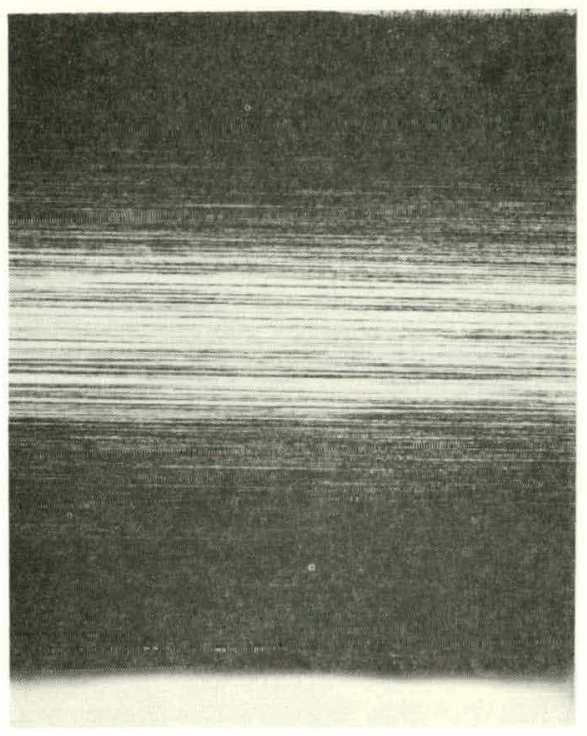

(a)

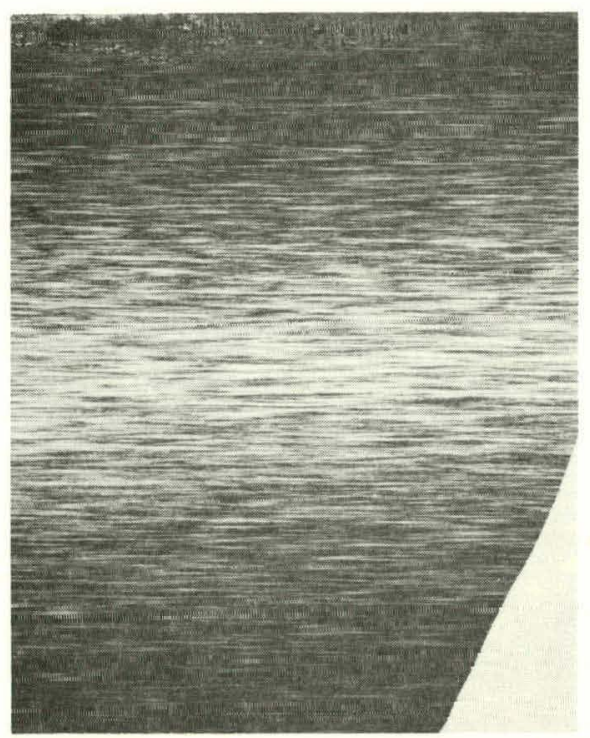

(c)
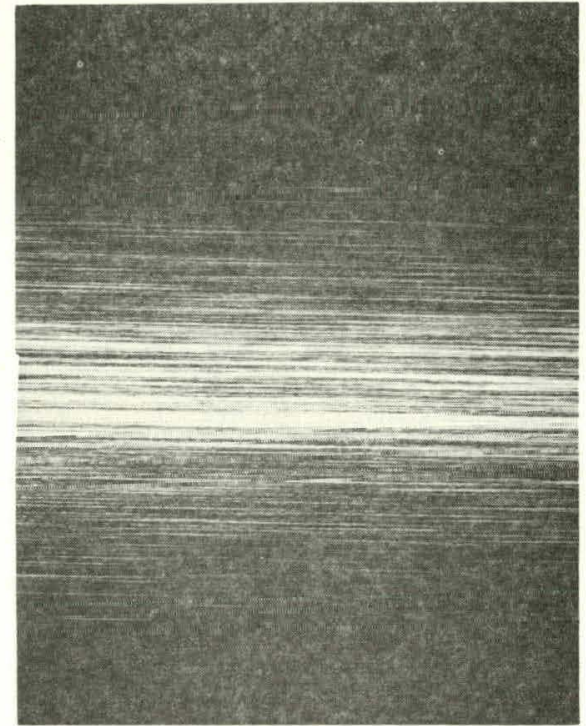

(b)

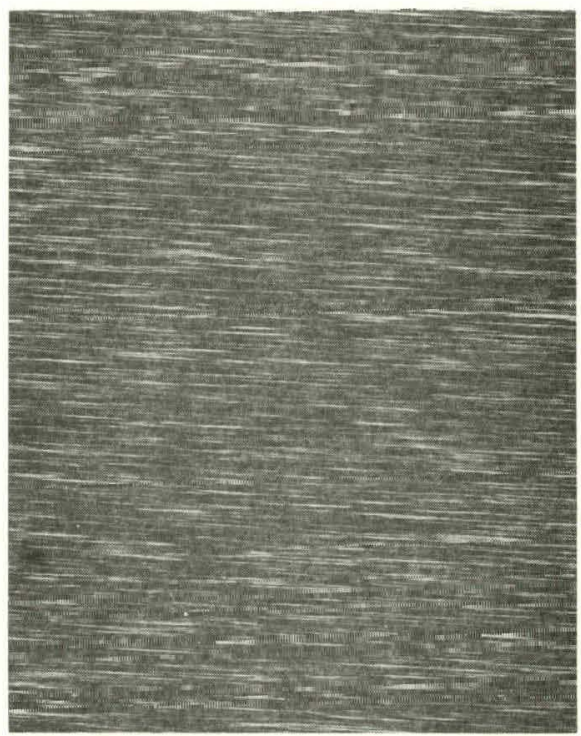

(d)

FIGURE 8. Photographs of Laser Diffraction Patterns from (a) 2 Microinch (b) 4 Microinch (c) 8 Microinch Lapped Surfaces and (d) Simulated Corrosion Deposit on Inside of Tube. Note that all are spread out in the horizontal direction due to focusing of cylinder lens. 


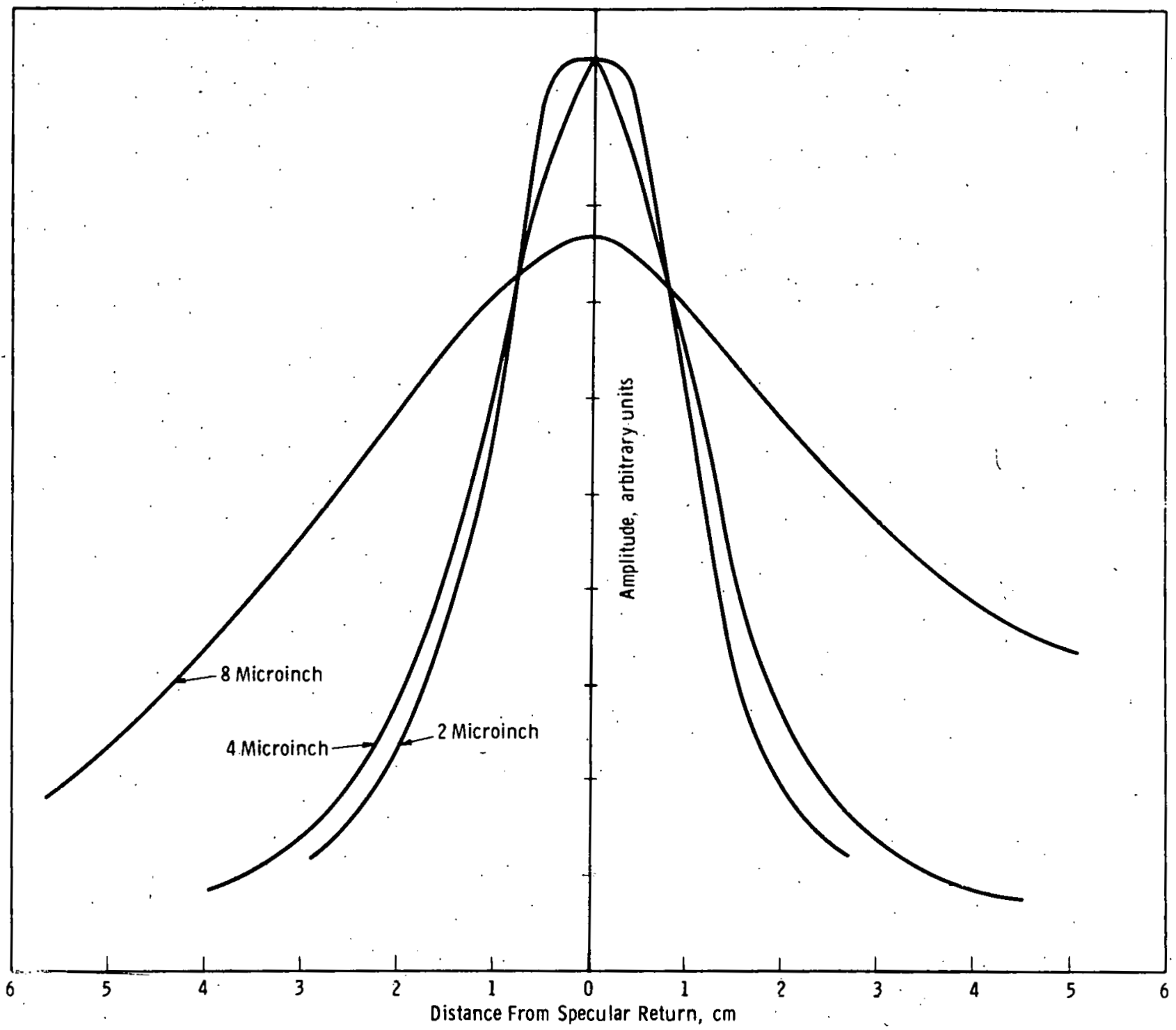

FIGURE 9, Densitometer Traces of Photographs in Figure 8a-c. 
a gaussian dependence on angle. From the angle $\Delta \theta_{1 / e}$ at which $I_{\text {scat }}$ drops to $1 /$ e of the peak, the ratio of the correlation distance to roughness can be calculated from

$$
\Delta \theta_{1 / \mathrm{e}}=4 \sigma / \mathrm{T}
$$

The correlation distance in the above equations is a parameter which represents the distance one can move a surface relative to itself and have the autocorrelation function of the surface drop to 1/e. Since the variable $(\mathrm{T} / \dot{\sigma})$ in $(8)$ and $(9)$ is a ratio between height and distance, the scattering described by ( 8 ) is determined by the slopes of the scattering centers on the surface. The assumptions used to derive (3) are that the roughness is on the order of a wavelength of the scattered radiation, and that the scattering angles are sma1l. For extremely. rough surfaces the behavior shown in Figure 9 extends to much higher angle scattering. Measurements in that case"would require moving the film plate closer to the surface to be measured. As will be seen below, this also changes the scale of the information contained in the diffraction pattern.

We have seen above how that measurements of the amplitude of the light scattered as a function of angle away from specular return provides information about the roughness of the sample surface. In the case of laser scattering an additional, bit of data is available, namely, the spatial frequency content of the speckle pattern of the diffracted light. Spatial frequency is a means of describing a distribulion of intensities, for example a series of light lines on a dark background. The term is in complete analogy to the customary notions of amplitude and frequency in the electromagnetic domain. For example, when an arbitrary amplitude function $A(x)$ of $x$ is represented by its spatial fourier tranșform:

$$
A(x)=\int_{-\infty}^{\infty} B(\omega) \exp (i \omega x) d \omega
$$


the quantity $w$ (which has the dimensions of length ${ }^{-1}$ ) is the spatial frequency and $B(\omega)$ is the spatial frequency distribution function for $A(x)$. If $A(x)$ is a Sinusoidal function of $x$, then the spatial frequency distribution is a delta function at $\omega=\omega_{0}$ :

$$
\begin{aligned}
& \text { If } A(x)=\cos \left(\omega_{0} x\right) \\
& \text { Then } B(\omega)=\pi / \omega_{0} \delta\left(\omega-\omega_{0}\right) \text {. }
\end{aligned}
$$

Note that any physically realizable amplltude transmission function can be described in terms of its spatial fourier transform.

Goldfischer ${ }^{5}$ has shown that the speckle pattern of laser light scattered by a diffuser has a power spectral density which can be calculated from the autocorrelation function of the illumination on the surface. If the surface consists of scatters with random phases distributed over the surface, the amount of power scattered into a spatial frequency region about a frequency $\omega, S(\omega)$, is proportional to the square modulus of the Fourier transform of the intensity distribution. In terms of the illuminating wavelength $\lambda$ and is the distance from the scattering surface to the recording plane, $R$, this dependence is

$$
S(\omega) \sim(\lambda / R)^{2}|\mathrm{Ft}[I(x)]|^{2}
$$

Thus the spatial frequencies in the diffraction pattern of laser light scattered from a surtace concaln Infuimation about the spatial frequencies of the scattering surface.

The experimental arrangement in Figure 7 was used to obtain photographs of the one dimensional scattering from the standard surfaces of nominal roughness of 2,4 , and 8 microinches as well as the diffraction pattern from a corrosion surface supplied by Bettis. These photographs, shown in Figure 8, consist of horizontal lines of variable spacing in the vertical direction. In order to determine the distribution of spatial frequencies in these lines, the transparencies were fourier analyzed with a coherent optical processor. The recordings of the fourier analysis were made photographically and the readout of 
the amplitudes of the fourier components was made by scanning the recordings of the fourier transform with a microdensitometer. The smoothed results appear in Figure 10 where the output of the microdensitometer is plotted as a function of spatial frequency of the photographic negative. It is clear from this figure that the spatial frequency content of the diffraction pattern is correlated in some manner with the properties of the surfaces. Curve A corresponds to the smoothese surface which should have the lowest spatial frequency content. Curves $\mathrm{B}$ and $\mathrm{C}$ correspond to progressively rougher surfaces and curve. $D$ is the distribution for the corrosion product deposition surface. The oscillations in amplitude near zero spatial frequency are noise introduced by the coherent optical processor and should be ignored. From these curves qualitative comparative behavior of the different surfaces can be obtained; quantitative information is not available because it is not clear at this time whether Goldfischer's analysis applies to the case of scattering by a uniformly reflecting surface with random heights and even if it does apply precisely what information about the surface is obtained from these measurements. These are matters which require further analysis beyond the scope of the present work.

In summary, the laser scattering technique allows a direct measure of the statistical slope distribution function from measurements of the intensity of the light scattered away from the specular return. The same photographic record which is used to obtain the above information can be Fourier transformed with an optical analog computer and additional comparative information related to the spatial frequency distribution of the scattering centers can be obtained: From these measurements a statistical description of the corrosion surface can be determined.

EASE OF APPLICATION OF THE TECHNIQUES

It appears from the experiments outlined above that hologram recording of the corrosion product deposits with a monocular microscope offers considerable advantage over conventional photography 
Curve $583555-c$

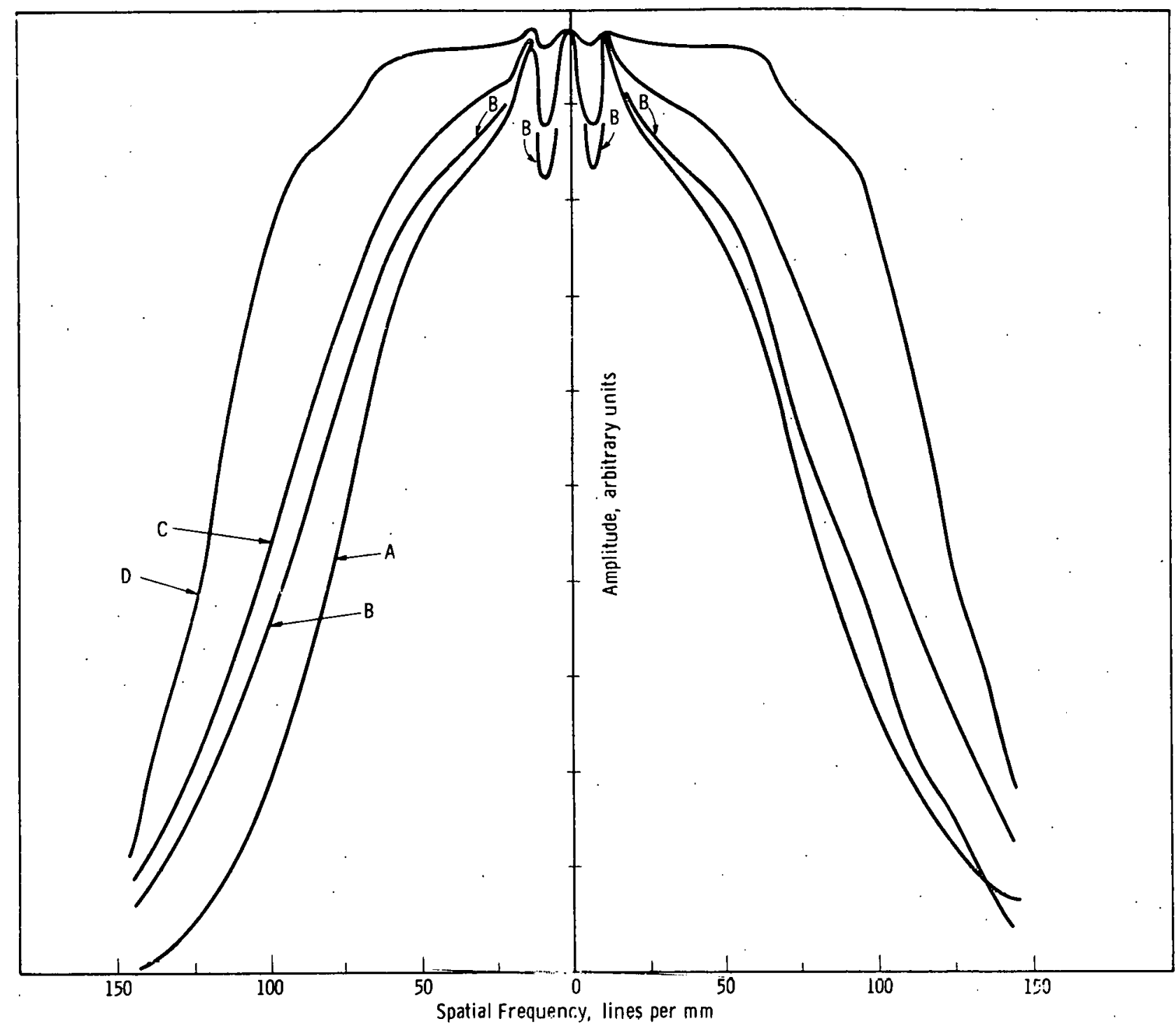

Figure 10. Spatial Frequencies of Figures 8 a-d as Determined by a Coherent Optical Processor. 
since the depth of field of the hologram reconstruction is considerably greater than that of a photograph. As a result, the hills and valleys in the surface can be recorded and the reconstruction from the hologram will contain images which can be refocussed at leisure. Holography offers the additional advantage of the possibility of recording contour information at the same time using double exposure holography with illuminating source moved between exposures or using an immersion cel1. Both American Optical ${ }^{8}$ and Bausch and Lomb ${ }^{9}$ have reported the development of hologram recording microscopes. Thus this technique may be availábile as a commercial item.

Roughness measurements by means of scattered laser light could be performed with essentially the same laser and optics as the holography. A slightly different optical system could be chosen using movable mirrors or alternatively a second laser could be employed. Alignment of the sample with respect to the optics is relatively uncritical so conventional handling devices would be adequate. This technique is actually simpler than the holographic recording since it involves simply a photographic recording of the coattcred liğlıL without the necessity of supplying any reference wavefront.

The precision of the contour method of holography can be assured by the measurement of a standard sample before each run to check the predicted slope sensitivity. In principle, however, a standard is not necessary since the sensitivity of the technique can be predicted from the parameters of the experiment.

The roughness measurements, at the present time, give only relative measurements. Therefore, information would have to be obtained on surfaces of known roughness for comparison.

\section{AUTOMATIC INTERPRETATION OF RESULTS}

Automatic interpretation of a hologram recording of the corrosion surface without the contours superimposed would be difficult although not impossible. Height information would be extracted by focussing on the portion of the image of interest. Projection of a real image on to a screen as is done in photogrammetric reduction of stereo pairs would not produce a three dimension image. 
Holograms with contour information built into them, on the other hand, could be interpreted automatically; two dimensional photographs of the contours could be scanned automatically, and the digital information on punched cards could be analyzed by computer to interpret the contours. For example, a photograph such as Figure $5 \mathrm{~d}$ would require only about 500 samples in the horizontal direction to provide 10 points per fringe.

Another advantage of analyzing photos with the contour information is that only a few gray scale elements are required since the contrast between the maximum and minimum of a fringe is well defined. A computer program for analyzing such a fringe system can be simple since the only requirement for measurement is to determine the position of the fringe relative to its neighbors. Using equation 7 , and the known geometrical parameters of the system, the height change per fringe can be calculated from the fringe spacing. A computer program for analyzing the 500 by 500 array of points for the distance between neighboring fringes would be simple when compared with a comparable program for analyzing stereo photographs photogrammetrically. Furthermore, since the information desired is coded in terms of fringes, much lower resolution of scanning and an accompanying reduction of computer memory allocation is required when compared with the accuracy and resolution required for feature extraction and measurement from stereo photographs. To obtain 500 points along a 5 inch trace requires only .010 iuch resolution. For mnst economical processing the gray scale should be kept to as few elements as possible; if contract does not vary across the photograph, perhaps the output could be quantized to two levels, black and white. The analyzing computer program would then only have to find the edges of the fringes and measure their distance from the neighboring fringe. In short, the hologram contour generation technique appears to offer the most promise for simplified automatic interpretation. of microphotographs.

Automatic readout of the roughness data similar to' that of Figure 4 can be performed with an array of photocells or solid state pholudetectors. Note that light is scattered over a considerable 
angle by the rougher surfaces; therefore photoconductive arrays 10 of low resolution can be ised to readout the desired intensities." The intensity as a function of angle could be read out on a series of meters as the ratios of voltage at different distances from specular or laternatively, the light distribution could be scanned with one photocell and the digitalized result analyzed with a computer. The spatial frequency data would best be recorded on photographic film for later optical processing. The coherent optical processor consists of simply a collimated source of laser light and a lens arranged so that the input photograph is placed one focal length from a lens. At a distance of one focal length on the other side of the lens, the spatial Fourier transform of the input photograph appears. The intensity of this transform can be recorded photographically or can be read out with a scanned photosensitive device.

\section{RECOMMENDATIONS FOR FURTHER RESEARCH}

Since the present work has shown the feasibility of using holographic techniques in the surface topography measurement problem, further work for developing the techniques to the point where they are useful in the actual measuring situation is needed. Furthermore, more research along the lines outlined below should be done both to improve the techniques whose feasibility has been demonstrated and to develop new inspection tools.

A research program should be carried on with the following goals.

1. Development of real time contour information by means of projection of fringes on the microscopic surface. Real time information of the type generated by the hologram contour generation experiments would allow virtually instantaneous analysis of the contour data by using videcon input to an on-line computer. With photographic recording of the results polaroid photography would allow immediate determination of the success of any given photograph. Holographic recording of the data would provide a permanent three dimensional record. 
2. Further development of the immersion technique of contour generation. This technique provides a record with contours which are more easily interpreted than those for oblique illumination. Construction of a double optical cell should allow the extension of this technique to higher magnifications than demonstrated in this paper.

3. Theoretical analysis of the spatial frequency distribution of diffraction patterns from rough surfaces. This analysis would answer the question of which parameter of the surface is responsible for the scattering behavior shown in Figure 10.

4. Investigation of pulsed laser holography as applied to the first two items. Determination of the parameters of a pulsed laser system appropriate to holographic microscopic recording.

5. Investigation of the possibility of other holographic geometries such as local reference beam holography. Use of the local reference beam technique would simplify the hologram recording geometry providing that the technique is capable of providing the required resolution.

6. Examination of other potential techniques such as automatic focussing.

RECOMMENDATIONS FOR A DEVELOPMENT PROGRAM

Development of both the holographic techinique and the laser scattering technique should proceed in two steps. First, an experimental laboratory model of each experiment should be set up in order to determine the actual engineering parameters such a type and stability of optics which will be required for the final design of an apparatus. Both the laser scattering and holographic studies could be done with a $100 \mathrm{mw}$ argon laser such as the RCA LD 2100. With the laser scattering experiment with photoelectric feedout, comparative data on various samples could be obtained.

Specific recommendations for the hologram microscope arrangement are difficult to make at the present time. It would appear that a real time contour fringe system would offer distinct advantages 
over the holographic generation of contours. It is not clear whether the three dimensional imaging and recording aspects of holography would be needed if the contour information could be recorded with fringe projection and conventional photography.

Since the fringe projection and two double exposure holography techniques convert height information into. low resolution contours, the photographic scanning apparatus and computer program for interpretation of the results will be the same for all systems. Therefore design of the computer program and purchase of the scanning apparatus.could be initiated at this time.

While automatic readout of the intensity of laser scattering as a function of angle from specular can be realized photoelectrically; with little trouble, the spatial frequency analysis requires the construction of a coherent optical processor. While this is not a complicated matter, construction of this apparatus should await the theoretical calculation outlined above to determine whether the spatial frequency spectrum contains desirable information about the surface roughness.

In conclusion, certain aspects of this study such as the laser scattering experiments and hologram recording of microscopic images can be applied to the roughness determination with relatively little further research. Application of other aspects such as the contouring should be deferred until research on fringe projection contouring is completed. 


\section{REFERENCES}

1. B. P. Hildebrand and K. A. Haines, J. Opt. Soc. Am. 57, 155 (1967).

2. T. Tsuruta, N. Shiotake, J. Tsujiuchi, K. Matsuda, Japan, J. App1. Phys. $\underline{6}, 661$ (1967).

3. M. Francon, "Optical Interferometry", Academic Press, New York (1966) p. 279.

4. S. Tolansky, "Surface Microtopography", Longmans, London (1960).

5. L. I. Goldfischer, J. Opt. Soc. Am. 포, 247 (1965).

6. P. Beckmann and A. Spizzichino, "The Scattering of Electromagnetic Waves .from Rough Surfaces", Macmillan, New York (1963).

7. S22 Microfinish Comparator -- Mfg. by Fairchi1d Development Labs Stamford Connecticut.

8. R. F. vanLigten, J. Opt. Soc. Am. 57. 564 (1967).

9. K. Snow and R. Vandewarker, J. Opt. Soc. Am. 57, 1406 (1967).

10. For example -- PIN Spot 10 photodetector: United Detector Technology, P. O. Box 2251 Santa Monica California.

11. J. Zelenka and J. Varner, App1 Opt. 7, 2107 (1968). 
Notice of Issuance to:

Ime :

r!:

spartment :

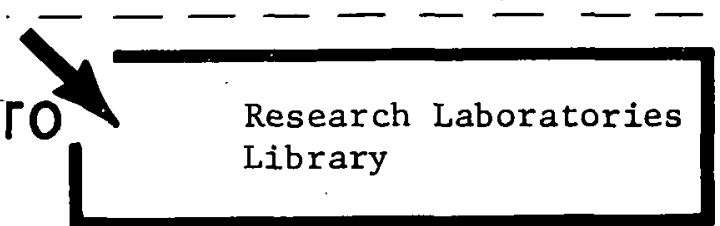

TDS- WERL-HOLOG- 1

Send Copy of Document to:

Plant

Dept.

Send TDS Card to:

Plant

Dept .

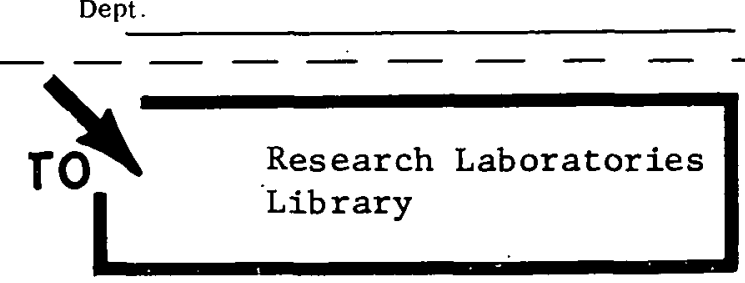

TDS- WERL-HOLOG- 1

Send Copy of Document to:

Plant

Dept.

Send TDS Card to:

Plant

Dept.

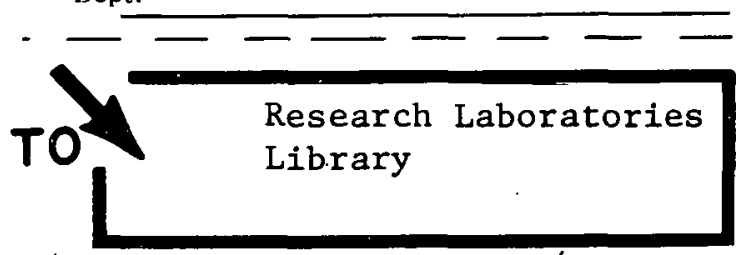

TDS-WERL-HOLOG-1

Send Copy of Document to:

Plant

ept.

TDS Card to:

Plant

Dept.
The TDS card is the basic tool for disseminating and retrieving Westinghouse technical information. Use this TDS card for sending notice of issuance of a report, memo, or paper to those who may wish to request a copy of the document by returning the stub cutout. Include a TDS card in each copy of the document. The $3 \times 5$ card cutouts provide for filing references in personal or group systems. For further information see Keyword Dictionary and Keyword Index and Abstracts issued by Research Laboratories. These are Proprietary Class 2 documents.
TDS- WERL-HOLOG-1

1ssuing Dept., Plant or Dlv. Research Laboratories

Proprietary slass: pages: 29 references: 11 Date: December 18, 1967

If govt. supported, contract number is -

LASER TECHNIQUES FOR APPLICATION TO SURFACE TOPOGRAPHY, G.B. Brandt

lasers, holography, topography, microscopy, scattering

Four laser techniques have been developed for the study of the topography and surface roughness of simulated corrosion sample surfaces. Two methods using holographic contour generation provide depth information with approximately 10 micron resolution. Two laser scattering experiments provide roughness data. Experimental results and recommendations. for further research are presented.

\section{TDS- WERL-HOLOG-1}

Issuing Dept. , Piant or Div. Research Laboratories

Proprietary class: pages: 29 references: 11 Date: December 18, 1967

If govt, supported, contract number is -

LASER TECHNIQUES FOR APPLICATION TO SURFACE TOPOGRAPHY, G.B. Brandt

lasers, holography, topography, microscopy, scattering

Four laser techniques have been developed for the study of the topography and surface roughness of simulated corrosion sample surfaces. Two methods using holographic contour generation provide depth information with approximately 10 micron resolution. Two laser scattering experiments provide roughness data. Experimental results and recommendations for further research are presented.

TDS- WERL-HOLOG-1 Issuing Dept., Plant or Div.

Research Laboratories Proprietary class: pages: 29 referénces: 11 Date: December 18, 1967

If govt. supported, contract number is -

LASER TECHNIQUES FOR APPLICATION TO SURFACE TOPOGRAPHY, G.B. Brandt

lasers, holography, topography, microscopy, scattering Four laser techniques have been developed for the study of the topography and surface roughness of simulated corrosion sample surfaces. Two methods using holographic contour generation provide depth information with approximately 10 micron resolution. Two laser scattering experiments provide roughness data. Experimental results and recommendations for further research are presented. 
Research

*W.E. Shoupp

J.W. Coltman

J.H. Bechtold (2)

*R. E. Fox

$*$ R.R. Heikes

*J.K. Hulm

*J. Swiss

*L. B. Kramer

*A.H.B. Walker

E.G.F. Arnott

R.D. Haun, Jr.

H.F. Ivey

I. Liberman

J.D. Feichtner

N.T. Melamed

E.P. Riede 1

K.B. Steinbruegge

E.W. Sucov

T. Isaacs

P. Buchhave

R. Johannes

L.A.C. Weaver

A. Khadj avi

G. Harkness

M. Gottlieb

$T$. Henningsen

R. Zollweg

J. Simpson

A. K. Rigler

T.P. Vog1

H.A. Unvala

D. Muss

L.G. Harper (3)

Hq. Mfg. Labs.

F.G. Hannd

Baltimore Aerospace

G. Tisdale
Aerospace Cambridge, Mass.

$$
\text { W. E. Horn }
$$

R. Wood

A. Sciola

Patent Dept.

R\&D Center

*E. Klipfel

Youngwood

A.I. Bennett

Bettis - NRTF

Idaho Falls, Idaho

R. Winchester

M. Linde

\section{Bettis}

S. Bohn

M. Krall (15)

Waltz Milis

J.C. Kelly, Jr.

Astronuclear

P. Gaal

A.P.D.

C.R. Taylor

W.D. Fletcher

$\vec{P}$. Cohen

*TDS CARD ONLY 